9 Galiè N, Hoeper MM, Humbert M, et al. Guidelines for the diagnosis and treatment of pulmonary hypertension. Eur Respir J 2009; 34: 1219-1263.

10 Kotecha S, Barbato A, Bush A, et al. Congenital diaphragmatic hernia. Eur Respir J 2012; 39: 820-829.
11 Cerro MJ, Abman S, Diaz G, et al. A consensus approach to the classification of pediatric pulmonary hypertensive vascular disease: report from the PVRI Pediatric Taskforce, Panama 2011. Pulm Circ 2011; 1: 286-298.

DOI: $10.1183 / 09031936.00114212$

\title{
The anti-IL-17A antibody secukinumab does not attenuate ozone-induced airway neutrophilia in healthy volunteers
}

\section{To the Editor:}

Within the lung the T-helper cell interleukin (IL)-17 (also referred to as IL-17A) is recognised as one of the principal cytokines that links the activation of T-lymphocytes to the chronic accumulation of neutrophils. It has been proposed that IL-17A is a relevant pharmacological target in inflammatory lung diseases with sustained mobilisation of neutrophils [1].

Secukinumab (AIN457; Novartis Healthcare Pvt. Ltd, Basel, Switzerland) is a recombinant high-affinity fully human

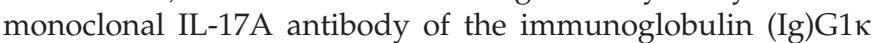
isotype, which binds to human IL-17A and neutralises the bioactivity of this cytokine [2].

Ozone exposure in healthy volunteers induces an acute and reproducible neutrophilic airway inflammation, which can be utilised as a pharmacological model to test anti-inflammatory drugs in early development [3-5].

We performed a single centre, phase II, double-blind, placebocontrolled, parallel-group study with an open label reference arm, investigating the ability of secukinumab to attenuate airway neutrophilia in induced sputum $24 \mathrm{~h}$ and $48 \mathrm{~h}$ following ozone exposure in healthy volunteers. Subjects underwent a $3 \mathrm{~h}$ ozone challenge, and induced sputum samples were collected $24 \mathrm{~h}$ and $48 \mathrm{~h}$ after the challenge. Subjects with an inflammatory response to ozone (as reflected by an increase in the total number of sputum neutrophils by at least $50 \%$ compared with the screening visit) were then randomised to receive either secukinumab (10 $\mathrm{mg} \cdot \mathrm{kg}^{-1}$ bodyweight) or placebo, in a double blind fashion administered as an infusion over 120 mins or to an open-label single-dose oral corticosteroid treatment (OCS, $50 \mathrm{mg}$ ) in the ratio of 2:1:1 (secukinumab:placebo:OCS).

The secukinumab/placebo treatment period consisted of a single dosing visit on day 8 followed by an ozone challenge 1 week later on day 15 and subsequent sputum inductions on day 16 and day 17. A third ozone challenge was performed on day 36 in the first nine subjects and on day 64 in the second nine subjects assigned to secukinumab or placebo. Subjects assigned to OCS treatment received a single dose treatment $1 \mathrm{~h}$ before the start of the ozone challenge on day 15. Sputum induction followed $24 \mathrm{~h}$ and $48 \mathrm{~h}$ after the challenge.
The Ethical Committee of Schleswig-Holstein, Germany approved the study and all subjects provided written informed consent.

The inhalation of $250 \mathrm{ppb}$ ozone and processing of sputum was performed as previously described [3-5].

Secukinumab concentrations in serum were determined by a competitive ELISA assay. Safety assessments and an assessment of immunogenicity to secukinumab were performed at various visits.

The study tested the null hypothesis of no difference between the secukinumab and placebo group in total number of neutrophils in induced sputum $24 \mathrm{~h}$ after ozone. Post hoc power assumptions using the observed standard deviation of $4.2 \times 10^{6} \cdot \mathrm{mL}^{-1}$ showed that 12 and 6 subjects in the secukinumab and placebo groups, respectively, provide $80 \%$ power to detect a difference of $5.5 \times 10^{6} \cdot \mathrm{mL}^{-1}$ using a two-sided test at $10 \%$ alpha. Standard ANCOVA methods, including baseline adjustment, were applied to neutrophil counts. Data were log-transformed to achieve a normal distribution. No alpha adjustments were made.

24 Caucasian subjects (19 males and five female with mean: age $40 \mathrm{yrs}$ and body mass index $24.2 \mathrm{~kg} \cdot \mathrm{m}^{-2}$ ) were enrolled in the study, of which 23 subjects completed the study. One subject was withdrawn for administrative reasons. The mean sputum neutrophil count at screening was $0.63 \times 10^{6} \mathrm{~mL}^{-1}$ and increased to $4.19 \times 10^{6} \mathrm{~mL}^{-1}$ after the baseline ozone challenge.

Regarding the primary end-point in this study, there was no significant difference observed between the treatment groups in the change from baseline in the total number of sputum neutrophils $24 \mathrm{~h}$ after the day 15 ozone challenge (day 16) (fig. 1). Sputum neutrophil counts were higher in secukinumaband OCS-treated subjects when compared with those receiving placebo $48 \mathrm{~h}$ after day 15 ozone challenge (day 17), with the latter reaching statistical significance. There was no significant effect of either secukinumab or placebo on the change from baseline in total number of neutrophils in induced sputum $24 \mathrm{~h}$ or $48 \mathrm{~h}$ after day 36 or day 64 challenges, with the exception of secukinumab-treated subjects $48 \mathrm{~h}$ after the day 64 challenge; driven by an unusually high number of sputum neutrophils in the placebo group. Evaluation of the change from baseline in the per cent of neutrophils did not reveal any treatment effects at any of the time points (data not shown). 


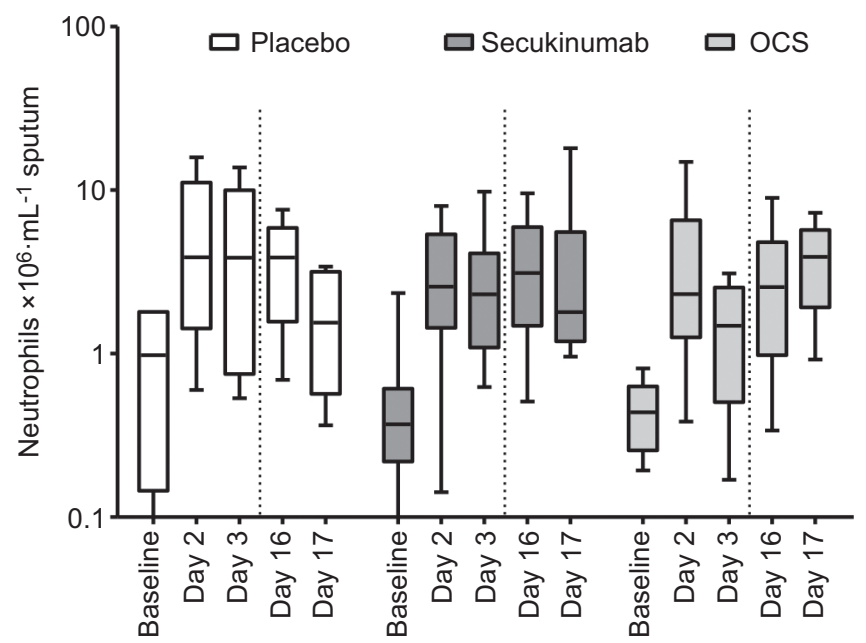

FIGURE 1. Box plot of the total number of sputum neutrophils. Sputum was induced at screening (baseline), $24 \mathrm{~h}$ and $48 \mathrm{~h}$ after pretreatment ozone challenge (days 2 and 3 , respectively), and $24 \mathrm{~h}$ and $48 \mathrm{~h}$ after post-treatment ozone challenge (days 16 and 17, respectively). The box represents the upper and lower quartiles, the horizontal line the median, and the whiskers the minimum and maximum values. The dashed line represents the timing of treatment (day 8 for placebo and secukinumab subjects and $1 \mathrm{~h}$ before ozone challenge on day 15 for oral corticosteroid (OCS) subjects).

The mean exposure as measured by area under the curve extrapolated to infinity (AUC0-inf) was 6,414 day $\cdot \mu \mathrm{g}^{-1} \cdot \mathrm{mL}^{-1}$ with a maximum concentration $\left(\mathrm{C}_{\max }\right.$ ) of $255 \mu \mathrm{g} \cdot \mathrm{mL}^{-1}$ was achieved at a median time of $2.15 \mathrm{~h}$ after the start of infusion. The mean half-life was 29.8 days. Serum concentrations of secukinumab declined rapidly until day 7 and then reduced more slowly over the subsequent days.

No unexpected events were observed and no subject had a serious adverse event. The overall incidence of the generally mild adverse events was similar in the secukinumab group $(n=8 ; 67 \%)$ and placebo group $(n=4 ; 67 \%)$. There were no clinically relevant changes in laboratory tests, ECGs, vital signs, spirometry or pulse oximetry, although a $97 \%$ (mean difference $3.25 \times 10^{6} \mathrm{~mL}^{-1}$ ) increase in blood neutrophils was noted $24 \mathrm{~h}$ after dosing in the OCS-treated group (no increase was observed in the other groups). No immunogenicity was observed in any of the subjects in this study.

We could not demonstrate a treatment effect of secukinumab on ozone-induced airway neutrophilia in this study. There are several potential explanations for these negative findings. It is possible that a higher dose of secukinumab might be required to fully neutralise local IL-17A production following ozone exposure. However, the dose used in this study was based on preclinical modelling and the doses currently used in other inflammatory disorders like psoriasis and rheumatoid arthritis [2].

Another possibility is that IL-17A inhibition alone is not sufficient to inhibit ozone-induced airway neutrophilia in humans. Repeated ozone exposure [6] and lipopolysaccharide exposure [7] in mice has been demonstrated to result in IL-17A dependent airway inflammation. The ozone model was chosen primarily due to the safety of the model and the non-invasive character of sputum induction. Nevertheless it cannot be ruled out that using, e.g. segmental endotoxin challenge, an inflammatory stimulus, which mimics bacterial infection and is associated with an IL-17A response in humans [8], would have yielded different results.

A comment is warranted regarding the non-effectiveness of OCS in the control group. We recently demonstrated an acute anti-inflammatory effect of OCS on sputum neutrophils $3 \mathrm{~h}$ after the end of the ozone challenge [3,4]. In the current study, unlike our previous studies, we studied induced sputum $24 \mathrm{~h}$ after the start of the ozone challenge and not $3 \mathrm{~h}$ post ozone challenge, based on nonclinical data suggesting that IL-17A is involved in a delayed $(24 \mathrm{~h})$ response to lipopolysaccharide [7]. The lack of attenuation in sputum neutrophils by OCS treatment may be related to a systemic neutrophilic leukocytosis $24 \mathrm{~h}$ after administration [9], potentially inducing a relative increase in the number of leukocytes in other compartments, such as the airway [10]. This assumption is supported by the observation that blood neutrophil counts in the six subjects receiving OCS increased by a mean of $97 \%, 24 \mathrm{~h}$ after dosing and ozone challenge, whereas the blood neutrophil counts in the other treatment groups were stable following ozone challenge.

\section{Anne Kirsten*, Henrik Watz*, Frauke Pedersen*, Olaf Holz*,\#, Rachel Smith ${ }^{\top}$, Gerard Bruin ${ }^{+}$,} Stephan Koehne-Voss ${ }^{+}$, Helgo Magnussen* and David A. Waltz ${ }^{\S}$ *Pulmonary Research Institute, Hospital Grosshansdorf, Center for Pneumology and Thoracic Surgery, Airway Research Center North, Member of the German Center for Lung Research, Grosshansdorf, and "Fraunhofer Institute for Toxicology and Experimental Medicine, Biomedical Research in Endstage and Obstructive Lung Disease Hannover (BREATH), Member of the German Center for Lung Research Hannover, Germany. "Novartis Institutes for BioMedical Research, Horsham, UK. ${ }^{+}$Novartis Institutes for BioMedical Research, Basel, Switzerland. ${ }^{\S}$ Novartis Institutes for BioMedical Research, Cambridge, MA, USA.

Correspondence: A. Kirsten, Pulmonary Research Institute at Hospital Grosshansdorf, Centre for Pneumology and Thoracic Surgery, Woehrendamm 80, D-22927 Grosshansdorf, Germany. E-mail: a.kirsten@pulmoresearch.de

Support Statement: Support for this study was provided by Novartis Pharmaceuticals Corporation, Basel, Switzerland.

Statement of Interest: Statement of interests for all the authors and the study itself can be found at www.erj.ersjournals.com/ misc/statements.dtl

Acknowledgements: The authors thank G Kretschmar, N. Stach, D. Beissel and M. Wendt (Pulmonary Research Institute, Grosshansdorf, Germany) for their excellent assistance in conducting the study, U. Zacharias (Novartism Nuremberg, Germany) for support during the study set-up and data collection period, and A. Thakur (Novartis Healthcare Pvt. Ltd, Hyderabad, India) for medical writing assistance. 


\section{REFERENCES}

1 Lindén A, Laan M, Anderson GP. Neutrophils, interleukin-17A and lung disease. Eur Respir J 2005; 25: 159-172.

2 Hueber W, Patel DD, Dryja T, et al. Effects of AIN457, a fully human antibody to interleukin-17A, on psoriasis, rheumatoid arthritis, and uveitis. Sci Transl Med 2010; 2: 52ra72.

3 Holz O, Tal-Singer R, Kanniess F, et al. Validation of the human ozone challenge model as a tool for assessing anti-inflammatory drugs in early development. J Clin Pharmacol 2005; 45: 498-503.

4 Holz O, Khalilieh S, Ludwig-Sengpiel A, et al. SCH527123, a novel CXCR2 antagonist, inhibits ozone-induced neutrophilia in healthy subjects. Eur Respir J 2010; 35: 564-570.

5 Kirsten A, Watz H, Kretschmar G, et al. Efficacy of the pan-selectin antagonist Bimosiamose on ozone-induced airway inflammation in healthy subjects - a double blind, randomized, placebocontrolled, cross-over clinical trial. Pulm Pharmacol Ther 2011; 24: 555-558.
6 Pichavant M, Goya S, Meyer EH, et al. Ozone exposure in a mouse model induces airway hyperreactivity that requires the presence of natural killer T cells and IL-17. J Exp Med 2008; 205: 385-393.

7 Ferretti S, Bonneau O, Dubois GR, et al. IL-17, produced by lymphocytes and neutrophils, is necessary for lipopolysaccharideinduced airway neutrophilia: IL-15 as a possible trigger. I Immunol 2003; 170: 2106-2112.

8 Glader P, Smith ME, Malmhall C, et al. Interleukin-17-producing T-helper cells and related cytokines in human airways exposed to endotoxin. Eur Respir J 2010; 36: 1155-1164.

9 Fauci AS, Dale DC, Balow JE. Glucocorticosteroid therapy: mechanisms of action and clinical considerations. Ann Intern Med 1976; 84: 304-315.

10 Nguyen LT, Lim S, Oates T, et al. Increase in airway neutrophils after oral but not inhaled corticosteroid therapy in mild asthma. Respir Med 2005; 99: 200-207.

DOI: 10.1183/09031936.00123612

\section{Cough frequency in health and disease}

\section{To the Editor:}

Chronic cough is a difficult clinical problem, partly because there is an absence of well-validated means to assess cough $[1,2]$. We have previously reported that the semi-automated computerised Leicester Cough Monitor detects cough accurately over $6 \mathrm{~h}$ and that cough frequency is increased in patients with chronic cough compared to controls [3,4]. There remains uncertainty on the performance of the system over $24 \mathrm{~h}$ and across the range of expected cough frequency in larger populations. We set out to address these questions in healthy adult volunteers and adult volunteers with respiratory disease.

44 healthy volunteers were recruited from those responding to a poster advertisement. All reported no current respiratory symptoms, were nonsmokers with a $<5$ pack-yr past smoking history and had normal spirometric values, methacholine airway responsiveness and induced sputum inflammatory cell counts. 78 patients with respiratory disease were recruited from respiratory clinics. The diagnostic criteria for the conditions have been described previously [5]. Six current smokers and four males taking angiotensin converting enzyme inhibitors (ACEi) were also recruited. The study was approved by the Leicestershire, Northampton and Rutland Research Ethics Committee.

All volunteers underwent spirometry and those with normal spirometry had a methacholine inhalation test using the tidal breathing method. The Leicester Cough Monitor (iRiver iFP-799 mp3 device; iRiver Europe $\mathrm{GmbH}$, Eschborn, Germany and Sennheiser MKE 2-5 field microphone; Sennheiser electronic $\mathrm{GmbH} \&$ Co. KG, Wedemark, Germany) was attached and recordings obtained and analysed as previously described [6].

The validity of the Leicester cough algorithm was assessed by randomly selecting 20 recordings (eight healthy volunteers, 12 patients with respiratory disease) which were analysed manually [3] by a blinded observer and then by the Leicester cough algorithm. The patients with respiratory disease had the following diagnoses: unexplained chronic cough $(n=6)$, asthma $(n=3)$, eosinophilic bronchitis $(n=2)$ and chronic obstructive pulmonary disease $(n=1)$. A capsaicin cough challenge was performed after removal of the cough monitor [5]. Sputum was induced and processed as described previously [7]. Patients with respiratory diseases completed the Leicester Cough Questionnaire (LCQ) [8]. Patients also completed a 100-mm cough Visual Analogue Score (VAS) [3].

Coughs per $24 \mathrm{~h}, \mathrm{C} 2$ and C5 (concentration of capsaicin required to elicit two and five coughs, respectively) were log normally distributed and were log transformed prior to analysis. An unpaired t-test was used to compare means between groups. Agreement was assessed by the intra-class correlation coefficient. Correlations between variables were analysed using the Pearson correlation coefficient for normally distributed data and Spearman's rank correlation coefficient for non-parametric data. Statistical analysis was performed using SPSS (version 16; SPSS Inc., Chicago, IL, USA).

The geometric mean $(\operatorname{logSD})$ manual and Leicester cough algorithm counts per $24 \mathrm{~h}$ in 12 patients with conditions associated with cough and eight healthy volunteers was 467 (0.32) versus $446(0.32)$ and $16(0.7)$ versus 22 (0.4), respectively. There was stronger agreement as assessed by the intra-class correlation coefficient between manual and counts in patients (0.98) than in healthy volunteers (0.85). The sensitivity and specificity, respectively, of the automated system was $83.8 \%$ and $99.9 \%$ in patients and $82.3 \%$ and $99.9 \%$ in healthy volunteers.

In the total population of healthy adults the geometric mean $(\log \mathrm{SD})$ number of coughs per $24 \mathrm{~h}$ was 18.6 (0.5). Females coughed more than males (geometric mean (SD) 29.5 (0.4) versus 8.3 (0.5); mean difference 3.5-fold; 95\% CI 1.9-6.8; $\mathrm{p}<0.001$ ). There was no correlation between 24 -h cough frequency and C2 $(r=-0.08)$ or C5 $(r=-0.03)$. Daytime cough counts (08:0022:00 h) were higher than night-time cough counts and hourly cough frequency tended to be higher in early morning and mid-afternoon. Cough frequency was not related to body mass index $(r=0.08)$ or age $(r=0.12)$. 